\title{
Dissipative transport across a parabolic barrier से
}

\author{
Helmut Hofmann \\ Physik-Department, TU München, James Franckstraße, W-8046 Garching, FRG \\ and \\ Gert-Ludwig Ingold \\ Fachbereich Physik, Universität-GHS Essen, W-4300 Essen I, FRG
}

Received 8 February 1991; revised manuscript received 23 May 1991

We suggest quantal diffusion coefficients for Kramers' transport equation and discuss the dynamics of fluctuations in the neighborhood of a barrier, considered generic for an instability of a system's response to an external perturbation.

\section{Introduction}

Instabilities play an important role in many parts of physics. Often they are treated within a mean field approach, sometimes from static considerations. For the classical case, Kramers has shown [1] how a dynamical treatment of statistical fluctuations may modify the results. It was only in the 80's that one has begun to understand how to incorporate quantum effects (for a review see e.g. refs. [2,3]). As the common technique one uses the method of path integrals, usually adapted to propagation in imaginary time. The latter feature is a mathematical artifact. Indeed, there exist many physical problems for which a description with real time propagation is highly desirable. For instance, this will be the case whenever the unstable mode is accompanied by additional observable processes. A prime example is nuclear fission at finite excitation which allows the evaporation of light particles. As another example we may mention nuclear multifragmentation. Here, it is already the complexity of the underlying equation of motion - which is of Boltzmann-Landau type including a collision

* This work has been funded in part by the German Federal Minister for Research and Technology (BMFT) under the contract number 06-TM-711. term - which may prohibit feasible numerical solutions for imaginary times.

For the typical situation considered by Kramers, the decay through a barrier out of a local potential minimum, there exists a formulation with real time propagation, at least for not too small temperatures. In ref. [4] it has been shown that all quantum corrections can be obtained in this way which had previously been derived with imaginary time propagation. In ref. [4] path integrals are also used. This restricts direct applications to cases where the coupling is linear in the bath variables, and for which the heat bath can be approximated by a quadratic form. Practically all examples from nuclear physics do not fall into this category. We will show that essential features can be taken over into a more general description making explicit use of transport equations. More specifically, we will generalize the formulation of ref. [5] to the case of a negative stiffness. This completes the method of describing large scale motion within the locally harmonic approximation [6,5].

\section{The basic equations}

We have in mind to study harmonic motion in the neighborhood of an extremal point at $Q=0$. In ref. 
[5] differential equations for the first and second moments have been suggested. The corresponding transport equation for the Wigner function $d(Q, P$, t) reads

$$
\begin{gathered}
\frac{\partial}{\partial t} d(Q, P, t)=\left(-\frac{\partial}{\partial Q} \frac{P}{M}+\frac{\partial}{\partial P} C Q+\frac{\partial}{\partial P} \frac{P}{M} \gamma\right. \\
\left.+D_{\mathrm{qp}} \frac{\partial^{2}}{\partial Q \partial P}+D_{\mathrm{pp}} \frac{\partial^{2}}{\partial P \partial P}\right) d(Q, P, t)
\end{gathered}
$$

provided we neglect possible initial correlations.

All transport coefficients appearing here can in the end be traced back to the system's response to an external perturbation. Typically the response function $\chi_{Q Q}(\omega)$ will have the following structure, when calculated within a self-consistent approach (see e.g. refs. $[6,7])$ :

$\chi_{Q Q}(\omega)=\chi(\omega) /[1+k \chi(\omega)]$.

Here, $k$ is some effective coupling parameter, and $\chi$ measures the local response of the intrinsic degrees of freedom to a variation of the collective ones. This information is all we need about the intrinsic degrees of freedom.

Generally, the information contained in eq. (2) will be richer than the one in eq. (1). A differential form for the time evolution can at best be true after picking some particular mode $\omega_{1}$ of (2), defined by $\chi_{Q Q}\left(\omega_{1}\right)^{-1}=0$. The transport coefficients for average motion, namely inertia $M$, friction $\gamma$ and stiffness $C$, can then be introduced by approximating the true response $\chi_{Q Q}(\omega)$ in the neighborhood of $\omega_{1}$ by one of a damped oscillator (see refs. [6,5]). Thus we replace the correct dispersion relation $1+k \chi(\omega)=0$ by

$-M\left(\omega_{1}\right) \omega^{2}-\mathrm{i} \gamma\left(\omega_{1}\right) \omega+C\left(\omega_{1}\right)=0$.

From here on the $\omega_{1}$ is kept fixed. Then the transport coefficients do not depend on frequency in true sense, but they do contain information about the actual dynamics. As we will discuss below, they even exemplify non-markovian features (cf. ref. [7]).

In ref. [5] the diffusion coefficients were defined through the quantal equilibrium fluctuations:

$D_{\mathrm{pp}}=(\gamma / M) \sum_{\mathrm{pp}}^{\mathrm{eq}}, \quad D_{\mathrm{qp}}=C \sum_{\mathrm{qq}}^{\mathrm{eq}}-(1 / M) \sum_{\mathrm{pp}}^{\mathrm{eq}}$.

They can be expressed in terms of $\chi_{Q Q}(\omega)$, again, by simply exploiting the fluctuation dissipation theorem (see e.g. ref. [8]):

$$
\begin{aligned}
\Omega & =\frac{1}{M \hbar} \sum_{\mathrm{pp}}^{\mathrm{eq}} \\
& =\frac{1}{M \hbar} \int_{-\infty}^{\infty} \frac{\mathrm{d} \omega}{2 \pi} \operatorname{coth}\left(\frac{\omega}{2 T}\right) \omega^{2} \chi_{Q Q}^{\prime \prime}(\omega), \\
A & =\frac{M}{\hbar} \sum_{\mathrm{qq}}^{\mathrm{eq}}=\frac{M}{\hbar} \int_{-\infty}^{\infty} \frac{\mathrm{d} \omega}{2 \pi} \operatorname{coth}\left(\frac{\omega}{2 T}\right) \chi_{Q Q}^{\prime \prime}(\omega) .
\end{aligned}
$$

For later convenience we have introduced two quantities, following a notation of refs. $[9,4]$. They have the following simple high temperature limit: $\hbar \Lambda=$ $T(M / C)$ and $\hbar \Omega=T$.

In ref. [5] only stable modes (with $C>0$ ) had been considered. In this case the $\Sigma_{\mu \nu}^{\mathrm{eq}}$ can be interpreted physically in the usual sense. However, there does exist an analytical continuation to the case with $C<0$. Still the integrals appearing in eqs. (5) and (6) can be calculated, once the pole approximation for the $\chi_{Q Q}(\omega)$ has been made. In this sense our more general treatment becomes identical to the calculation of Ingold [4]. For the integral in (5) it is of course necessary to apply some regularizing methods, as in the case of the stable modes. Here, we do not want to discuss questions about possible choices of the cutoff. Later on we will concentrate on an example for which only $A$ will appear.

For negative $C$, the solutions of (3) are purely imaginary: $\omega_{1}^{ \pm}=\mathrm{i} z_{ \pm}$, with

$z_{ \pm}=\omega_{\mathrm{b}}\left(-\eta \pm \sqrt{1+\eta^{2}}\right)$,

and

$\eta=\gamma / 2 \sqrt{M|C|}, \quad \omega_{\mathrm{b}}=\sqrt{|C| / M}$

being the dimensionless damping rate and the barrier frequency, respectively.

Before we move on to apply these results we would like to add some remarks on the possibly non-markovian nature of our approach. According to ref. [7] there are cases for which the average motion is described correctly by the common differential form of the equations of motion. This is true if the dissipative part of $\chi_{Q Q}(\omega)$ is of lorentzian type - in which case it has just two poles. Recently this has been demonstrated in ref. [10] to be a typical behaviour of isoscalar modes at higher temperatures. For such a form expression (6) can be evaluated without further ap- 
proximations. Through the regularization necessary for (5) the behaviour at very short times (comparable to one over the cut-off frequency) is modified. This alters the extent of a possible "non-markovian" structure, which otherwise is decided essentially by the reduction of the response function $\chi_{Q Q}(\omega)$ to the one of the damped oscillator. Of course, this approach only makes sense if the quantities $\Omega$ and $A$ evaluated in this way turn out to be physically meaningful. For our application we will have to come back to this question below.

So far we have not addressed the question of possible initial correlations between intrinsic and collective degrees of freedom. At not too low temperatures, such correlations will die out after some (macroscopically) small time. The same is true for the corrections which arise due to neglecting the poles of (2) at higher frequencies. Therefore, we claim the differential form of our basic equations to represent adequately the true situation. It is reassuring that our solutions for the second moments turn out identical to the ones derived in ref. [9] by applying path integrals to a model of coupled oscillators previously referred to, provided one takes care about the restrictions mentioned. As a matter of fact, in this letter we are going to use our equations only for examples which do involve the long time behaviour. We shall begin by looking at the transmission factor.

\section{Transmission factor}

We consider the transmission factor in a time dependent picture where "penetrability" has to be defined as

$$
\begin{aligned}
\Pi & =\lim _{t \rightarrow \infty} \int_{0}^{\infty} \mathrm{d} Q n(Q, t) \\
& =\lim _{t \rightarrow \infty} \int_{0}^{\infty} \mathrm{d} Q \int_{-\infty}^{\infty} \mathrm{d} P d(Q, P, t) .
\end{aligned}
$$

This is identical to the probability of finding the system to the right of the barrier at $t \rightarrow \infty$ if it had started at the left at some initial time. For a gaussian solution all integrations necessary can be carried through. For reasons of simplicity, in this letter we just look at the case where the system starts at $Q_{0}, P_{0}$ precisely, which means setting the initial widths of the distribution equal to zero. One then obtains:

$\Pi=\frac{1}{2} \operatorname{erfc}\left(\frac{-z_{-} Q_{0} M+P_{0}}{\sqrt{2 M \hbar\left[-\left(z_{-}\right)^{2} A+\Omega\right]}}\right)$.

A result of this type had been published previously in ref. [11]. This is seen by looking at their eq. (14), putting the initial fluctuations [called $\Gamma_{i}(t=0)$ ] equal to zero, and correcting for a typing error (the $\Omega_{r}^{2}$ should obtain a minus sign). The only difference is found in the new and correct choice of the diffusion coefficients (4). As for the transport equation itself we have to identify $\Delta_{\mu \nu}$ with $D_{\mu \nu}$. Considering these facts, the general case of a broad initial distribution is readily deduced.

In ref. [11] an extended discussion can be found of the nature of quantum effects contained in ref. [10]. We just mention the fact that for non-dissipative systems our result reduces to that of the Schrödinger equation. As for diffusion, in ref. [11] the high $T$ limit was taken.

\section{Quantum version of Kramers' stationary solution}

Kramers [1] had found that for $C<0$ eq. (1) has a stationary solution with non-vanishing flux. (Here, we have in mind the case of spatial diffusion.) In ref. [12] a connection to the time dependent solution was established: Integrating the latter over time the resulting expression "relaxes" into that of Kramers (as function of $Q$ ). This feature sheds some light on the physical nature of this stationary solution: It is meaningful for some statistically averaged processes only, and it can be valid at best after some initial time has passed. Within his analytical model, Ingold [4] was able to find the quantal extension of Kramers' form.

He did not look at a transport equation, but also in his treatment a long time limit had to be performed. With the parameters introduced above the new solution reads

$$
\begin{aligned}
& d_{I}(Q, P)=\zeta_{I}^{\prime} \exp \left[-\frac{M}{2 \hbar}\left(\frac{Q^{2}}{\Lambda}+\frac{P^{2}}{\Omega M^{2}}\right)\right] \\
& \times \int_{-\infty}^{P / M-a Q} \mathrm{~d} s \exp \left(-\frac{M}{2 \hbar\left(-\Lambda a^{2}-\Omega\right)} s^{2}\right),
\end{aligned}
$$


with $a=-\Omega /\left(A z_{+}\right)$. (For the following the normalization coefficient $\zeta_{I}^{\prime}$ will be immaterial.) By mere differentiation one may easily check that (11) does indeed solve eq. (1).

\section{Time delay through quantal diffusion}

Of considerable interest is the average time the system spends in the barrier region. In ref. [13] this time had been estimated from Kramers' stationary solution for the case of nuclear fission. We may just repeat the same steps here for the quantum case. To have one specific example, let us calculate the time the system needs to pass from saddle to scission. According to ref. [13] it can be defined as

$\bar{t}=\frac{1}{j_{I}} \int_{0}^{Q_{\mathrm{sc}}} \mathrm{d} Q n_{I}(Q)$

with the (constant) current given by

$j_{l}=\int_{-\infty}^{\infty} \mathrm{d} P \frac{P}{M} d_{I}(Q, P)$.

In a different context for such a time the notion "dwell time" had been used (see e.g. ref. [14]. The density $n_{I}(Q)$ is obtained similarly, integrating out the momentum without the velocity factor. The final result can be written as

$$
\begin{aligned}
\bar{t}= & \left(2 / \omega_{\mathrm{b}}\right)\left(\eta+\sqrt{1+\eta^{2}}\right) \\
& \times \mathscr{R}\left(\sqrt{M \omega_{\mathrm{b}}^{2} Q_{\mathrm{sc}}^{2} / 2 T} \sqrt{T /(-\Lambda) \hbar \omega_{\mathrm{b}}^{2}}\right) .
\end{aligned}
$$

The function on the right of (14) is defined as

$\mathscr{R}(x)=\int_{0}^{x} \exp \left(y^{2}\right) \mathrm{d} y \int_{y}^{\infty} \exp \left(-z^{2}\right) \mathrm{d} z$

(c.f. ref. [15]). Its form is plotted in fig. 1. In eq. (14) the argument $x$ of $\mathscr{R}(x)$ has been factorized like $x=x_{\mathrm{cl}} g_{\mathrm{qu}}$. This form makes apparent the quantum corrections to the case of the high temperature limit studied in refs. [13,12]. The first factor, $\sqrt{M \omega_{\mathrm{b}}^{2} Q_{\mathrm{sc}}^{2} / 2 T}$, simply is determined by the ratio of the difference of the potential energy between saddle and scission to the temperature. The second one, $g_{\mathrm{qu}}$, can be evaluated with the help of (6). With the response function $\chi_{Q Q}(\omega)$ approximated by the one of

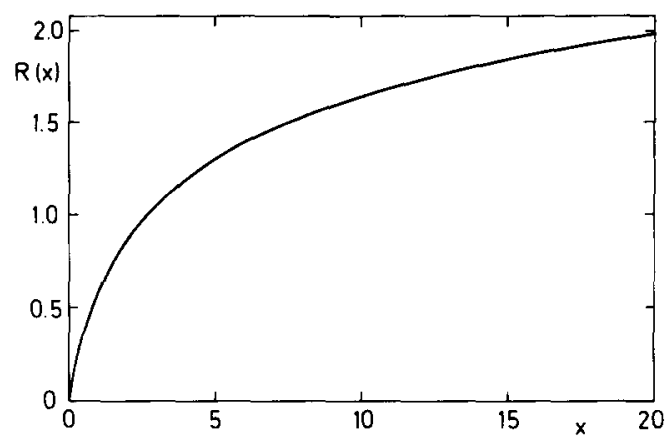

Fig. 1. Rosser's function $\mathscr{R}(x)$.

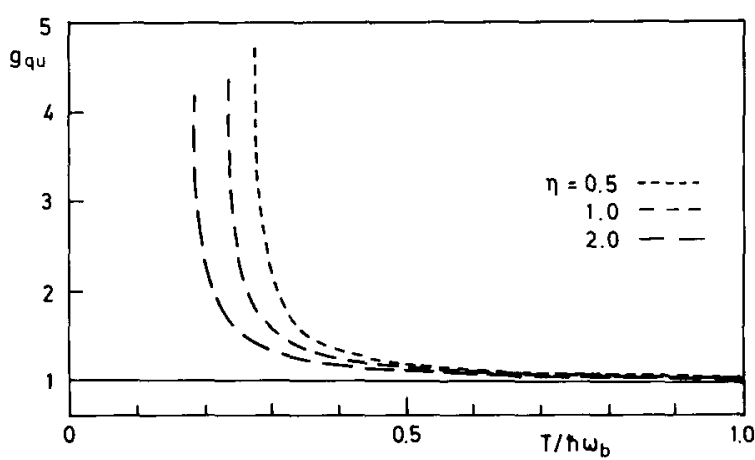

Fig. 2. The quantum correction factor $g_{\mathrm{qu}}$ as function of $T / h \omega_{\mathrm{b}}$ for three typical values of $\eta$.

the damped oscillator [ recall eq. (3) with $C<0$ ] one gets, after performing a contour integration and summing up all poles, and by introducing $\Psi$ as the logarithmic derivative of the gamma function:

$$
\begin{aligned}
g_{\mathrm{qu}}^{-2} & =\frac{-\Lambda \hbar \omega_{\mathrm{b}}^{2}}{T} \\
& =1+\frac{\hbar \omega_{\mathrm{b}}}{2 \pi T} \frac{1}{\sqrt{1+\eta^{2}}}\left[\Psi\left(1+\frac{\hbar \omega_{\mathrm{b}}}{2 \pi T}\left(\eta-\sqrt{1+\eta^{2}}\right)\right)\right. \\
& \left.-\Psi\left(1+\frac{\hbar \omega_{\mathrm{b}}}{2 \pi T}\left(\eta+\sqrt{1+\eta^{2}}\right)\right)\right] .
\end{aligned}
$$

The quantity $g_{\mathrm{qu}}$ is plotted in fig. 2 as a function of a dimensionless temperature $\tau_{\text {tem }}=T / \hbar \omega_{\mathrm{b}}$. At some small values the factor $g_{\text {qu }}$ tends to infinity. Possibly, this restricts the domain of validity of our approach. Indeed, at these temperatures the $\Lambda$ becomes zero indicating that the system ceases to behave diffusive. For higher temperatures we observe deviations from unity up to $\tau_{\text {tem }} \geqslant 0.5-1$. From the behaviour of Ros- 
ser's function in fig. 1 we see that this effect is to increase $\bar{t}$ due to quantum effects. The size of $\hbar \omega_{\mathrm{b}}$ depends on the deformation chosen and on its parametrization. For fission typical values are of the order of $1 \mathrm{MeV}$. For fusion [16] they would be a few times bigger.

\section{Discussion}

We have demonstrated how the computation of the diffusion coefficients of ref. [5] within a locally harmonic approximation can be extended to the barrier region. For such a situation we have studied two examples which can be treated analytically. The first one involves a truly time dependent picture. The second one deals with the stationary situation exploited already by Kramers.

As a quantitative application, we have taken the case where only the "generalized fluctuation" of $Q$ appears. For a full evaluation of both diffusion coefficients we would need the one in $P$ as well, see (4)(6), where regularization procedures had to be performed. It turned out that for this particular example quantum effects are to be expected at quite low temperatures only. This is in distinction to computations of diffusion coefficients for unstable modes. Indeed, take figs. 2 and 3 of ref. [5]. Sizable quantum effects can be observed up to temperatures of $2 \mathrm{MeV}$. From fig. 3 we see that the same effect is to be expected for stable modes: just extrapolate down to $C=0$.

The observed difference to the results for $\bar{t}$ is to be attributed to the fact that in $\bar{t}$ the quantity $\Omega$ does not appear explicitly. Indeed, for stable modes its value is known to be influenced by quantum effects up temperatures higher than the corresponding ones for $A$. For a propagation across a barrier the natural counterpart is the momentum distribution, say as given by Kramers stationary solution. Indeed, the mean kinetic energy and its variance can be evaluated from (11) completely analogously to the procedure of ref. [13]. For lack of space we will not present these results here. Of course, in the classical limit they can be shown to turn into those of ref. [13]. Just at which temperature this transition will occur requires a more careful computation of $\Omega$, which we would like to defer to later publications.

\section{Outlook}

The method suggested here to define and compute diffusion coefficients can be applied to several problems.

First of all we need to mention nuclear fission which is to be treated as a large scale motion using the propagator method (see e.g. refs. $[5,6]$ ). The transmission coefficient (10) might be used for fusion reactions (see ref. [17] and c.f. ref. [11]). (Recall that according to ref. [6] the temperature may play a less stringent role.) If non-linear effects turn out important, here also propagators could be introduced.

As indicated above, to derive the transport equation (1) all one needs as the basic ingredient are the response functions. They can be calculated whenever the proper equations for average motion are known. In this sense applications to equations of Boltzmann-Landau type or the ones of hydrodynamics are possible. Indeed, the authors of ref. [18] have begun to study the first example. This is a generalization of the treatment of instabilities of nuclear matter with response functions, given in ref. [19]. Similar instabilities supposedly happen in certain stages of the time development of the quark-gluon plasma. It would be most interesting to study this problem, too.

Furthermore, there remain a few other theoretical problems. First, there is the very intriguing question of what happens at lower temperatures. On decreasing $T$ the equations will cease to be of a differential nature. There is also still the problem of the decay rate for fission. The alert reader may have already noticed that this quantity has been missing in what has been said so far. Indeed, for a description with real time propagation, to date this problem has been solved only within the path integral method. Whether or not this will be possible by using the transport equation (1) will be discussed in a subsequent paper.

\section{Acknowledgement}

One of use (G.-L.I.) would like to thank H. Grabert for fruitful discussions on the problems of dissipative quantum systems. 


\section{References}

[1] H.A. Kramers, Physica 7 (1940) 284.

[2] P. Hänggi, J. Stat. Phys. 42 (1986) 105.

[3] P. Hänggi, P. Talkner and M. Borkovec, Rev. Mod. Phys. 62 (1990) 251.

[4] G.-L. Ingold, Thesis, Universität Stuttgart (1988).

[5] H. Hofmann, R. Samhammer and G. Ockenfuß, Nucl. Phys. A 496 (1989) 269.

[6] H. Hofmann, Phys. Scr. T32 (1990) 132.

[7] H. Hofmann, R. Samhammer and S. Yamaji, Phys. Lett. B 229 (1989) 309.

[8] D. Forster, Hydrodynamic fluctuations, broken symmetry and correlation functions (Benjamin, 1975).

[9] H. Grabert, P. Schramm and G.-L. Ingold, Phys. Rep. 168 (1988) 115
[10] H. Hofmann, S. Yamaji and A.S. Jensen, preprint (1991).

[11 ] H. Hofmann and R. Samhammer, Z. Phys. 322 (1985) 157.

[12] J.R. Nix, A.J. Sierk, H. Hofmann, F. Scheuter and D. Vautherin, Nucl. Phys. A 424 (1984) 239.

[13] H. Hofmann and J.R. Nix, Phys. Lett. B 122 (1983) 117.

[14] E.H. Hauge and J.A. Støvneng, Rev. Mod. Phys. 61 (1989) 917.

[15] J.B. Rosser, Theory and application of $\int_{0}^{z} \exp \left(-x^{2}\right) \mathrm{d} x$ and $\int_{0}^{z} \exp \left(-p^{2} y^{2}\right) d y \int_{0}^{y} \exp \left(-x^{2}\right) \mathrm{d} x$ (Mapleton House, New York, 1948).

[16] P. Fröbrich, private communication.

[17] F.L. Canto, Nucl. Phys. A 491 (1989) 337.

[ 18 ] H. Hofmann, D. Kiderlen and I. Tsekhmistrenko, preprint (1991).

[19] C.J. Pethick and D.G. Ravenhall, Ann. Phys. (NY) 183 (1988) 131. 